\title{
The impact of the accuracy of indicator diagrams on the heat release characteristics calculation, used in the diagnosis of marine diesel engine
}

\author{
Kazimierz Witkowski, ${ }^{1, *}$ \\ ${ }^{1}$ Gdynia Maritime Academy, Mechanical Faculty, ul. Morska 81-87, 81-225 Gdynia, Poland
}

\begin{abstract}
The paper analyzes the possibility to use the electronic type indicators in the diagnosis of marine engines. It has been shown that in-depth analysis of indicator diagrams would be useful - calculation of heat release characteristics. To make this possible, measuring indicated systems should meet a number of important requirements in or-der to ensure that they can be used for the diagnostic purposes. These includes: high precision sensors for the measurement of cylinder pressure, high speed and accuracy of measuring and recording of measured values. These also includes reliable determination of the top dead center piston (TDC). In order to demonstrate the impact of positional error TDC, simulation study was conducted in which indicated diagrams were used, obtained on a medium-speed four-stroke marine diesel engine type A25/30 and the low-speed two-stroke marine diesel engine type RTA76, Sulzer company.
\end{abstract}

\section{Introduction}

Nowadays many marine engine-rooms are additionally equipped with portable or stationary electronic indicators. Open (expanded) indicator diagrams are mainly analyzed - diagrams in a coordinate system $p=\mathrm{f}(\alpha)$ (cylinder pressure $\mathrm{p}$ variation as a function of crank angle $\alpha$ ). Indicated parameters are the essential complement to the standard measurement parameters and diagnostic inference can be more reliable $[1,2]$.

Measuring systems destined for indication should meet a number of important requirements to be met to ensure the possibility of their diagnostic use. These includes: high precision sensors for the measurement of cylinder pressure, high speed and accuracy of measuring and recording of measured values, a reliable determination of the top dead center piston (TDC). These and other important issues cylinder pressure measurement were highlighted in many publications [3-6]. Compliance with these requirements is particularly important when will be determined based on the indicator diagram of heat release characteristics for diagnostic purposes [7-10].

At old-design sea cargo ships with conventional equipment, marine engines diagnostics are still made on the basis of an analysis of changes in the value of the measured parameters of the engine $[7,11]$. Diagnostician is the ship's mechanic, who by analyzing changes in the measured parameters relative to their reference values, tries to determine the type of damage. Such action is subjective and usually inefficient, depending on the knowledge and experience of the marine engineer $[5,12$, $13]$.

\section{Direct and in-depth analysis of indicator diagrams in diagnostics}

Increasingly for indicated marine engines, it is being used the electronic type indicator, which includes: the combustion pressure sensors in each cylinder, the angular position sensor crankshaft and microprocessors system used for processing and visualization of the results of the measurements. This indicator is easy to use, characterized by high accuracy and fast developing results. This device gives results indicated as the average of dozen cycles. All essential values are displayed on the screen, and in addition an open indicator diagram is presented. Mean indicated pressure is automatically served beside other parameters, based on our numerical integration.

Figure 1 shows an example of an indicator diagram, obtained using the an electronic indicator. It is marked on the all important characteristic values. In addition to those indicated values in the figure, the mean indicated pressure and indicated power can be counted.

An important feature of electronic indicators is the ability to save the results in memory and play them after some time. This way, you can easily compare the graphs indicated which were performed at different times, which gives a fuller analysis capabilities [14-16]. This is particularly important during comparing the reference indicator diagram drawn in good technical condition of the engine with a graph removed after a longer period of operation; then are visible all deviations from the norm.

Figure 2 shows an example of one coordinate system $p=\mathrm{f}(\alpha)$ two graphs - curve 1 and curve 2 . The pressure value on the expansion curve $\left(p_{36}{ }^{\circ}\right)$ graph of measured

Corresponding author wika@am.gdynia.pl 
current (curve 2) is higher than the corresponding value for the standard graph (curve 1). This may be a prove of chronic working process, after-burning of the fuel during the expansion, e.g. due to incorrect fuel atomization. This state can also affect non-leak fuel injector nozzle or very poor fuel quality.

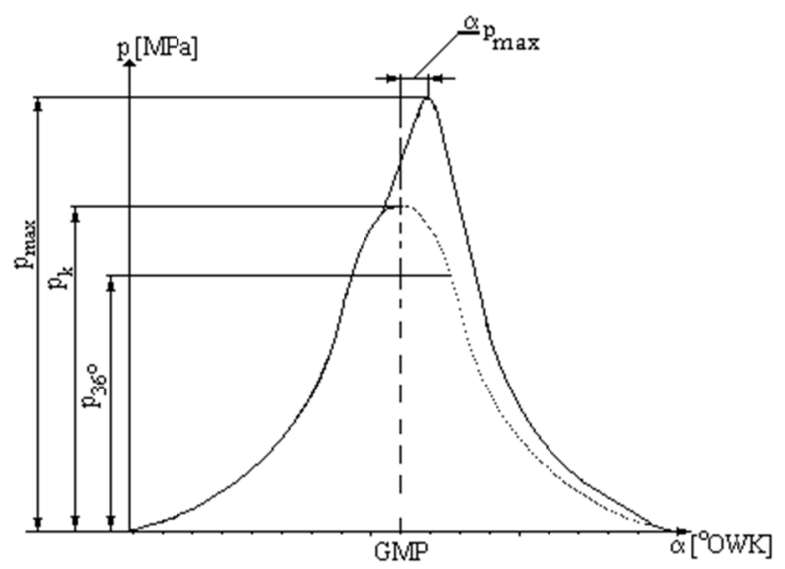

Fig. 1. Indicator diagram obtained from the electronic indicator and its characteristic values: $p_{\max }-$ the maximum combustion pressure, $p_{\mathrm{k}}-$ compression pressure, $\mathrm{p}_{36}{ }^{\circ}$ (or $p_{\text {exp }}$ ) - expansion pressure read 36 degrees of crankshaft rotation after TDC, $\alpha_{\text {pmax }}-$ angle after TDC obtain maximum pressure in the cylinder.

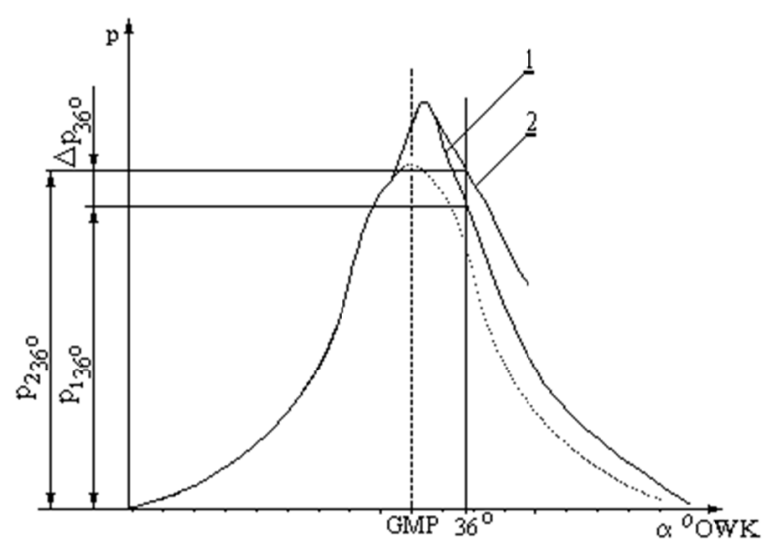

Fig. 2. Exemplary indicator diagram (1) and too high expansion pressure on the diagram (2).

The graph in Figure 3 shows that gain in pressure is too dynamic. Data from the graph allow to calculate the value of $\Delta p 2 / \Delta \alpha 2$. Based on a comparison of the measured $\Delta p 2 / \Delta \alpha 2$ with exemplary $\Delta p 1 / \Delta \alpha 1$, you can quantify the degree of damage. The cause of excessive dynamic rise pressure $-(\Delta p 2 / \Delta \alpha 2)>>(\Delta p 1 / \Delta \alpha 1)-$ may be premature fuel injection or ignition delay reduction: $\alpha_{\mathrm{zz} 2}<\alpha_{\mathrm{zz} 1}$, due to significant thermal overload the elements of the combustion chamber of the engine. A large value of $\Delta p / \Delta \alpha$ causes mechanical engine overload, especially bearings piston-connecting-rod system.

Ongoing monitoring and control of the mean indicated pressure $p_{i}$ provides a very accurate load current of each cylinder and also of the whole engine. On this basis we can determine whether the load status of each cylinder is the same or if there is too much spread of power between the cylinders [17-19]. In relation to the average of the instantaneous power of the cylinder, these differences should not exceed $\pm 2,5 \%$. With irregular engine regulation, mean indicated pressure pi in one or more cylinders will generally be lower than the average value.. There are at least several potential reasons for this: it may be due to defective regulation of the injection pump - too small dose of fuel on account of the piston-cylinder assembly leakage caused by poor technical conditions of injection equipment and air system supercharger.

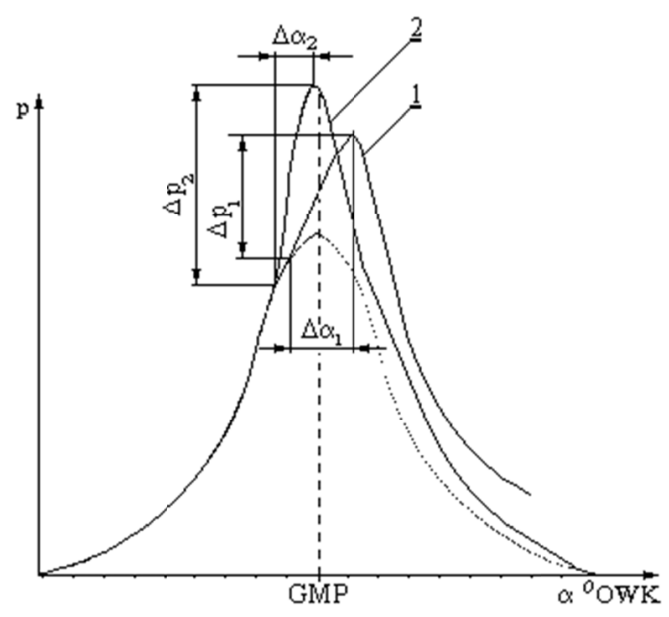

Fig. 3. Exemplary indicator diagram (1) and diagram with high value of $\Delta p / \Delta \alpha(2)$.

The direct analysis of the characteristics feature indicator diagram and changes of indicated parameter values does not always allow on the formulation of correct diagnostic conclusions. Even a few different defects can cause similar changes in the values of indicated parameters and shape indicator diagrams. Therefore this is recommended in-depth analysis of these graphs based on calculated heat release characteristics. Among other things, this is important in the diagnosis combustion process and of damage of injection system elements - injection pumps and injectors. Then, however, it is essential to cylinder pressure measurement accuracy - the accuracy of the indicator diagram. The analysis shows, that on the accuracy of the calculated heat release characteristics has a piston TDC location error on the indicator diagram.

\section{Analysis of the influence of selected factors on the accuracy of the indicator diagram}

A very significant impact on the accuracy of the determination of the heat release's characteristics, is the correct term for the indicator diagram obtained during the test engine, piston position placed in the top dead center (TDC). The main reasons of difficulty with accurately determine TDC are delays caused by crankshaft torsional vibrations, caused by indicated tunnels and cocks indicated, as well as due to nonuniformity of the crankshaft rotational speed $[3,4,9$, 
20]. It must be noted that between the kinematic angular position TDC piston and the TDC position shown on the indicator diagram, there is an error that will cause further errors calculated heat release characteristics. In [1, 3] stated that the error of determining TDC the amounting to $\pm 2 \%$, will cause distortion chart calculated heat release rate to about $\pm 12 \%$.

In many cylinder pressure measurement systems, errors occur, which are related to the "flow" of the reference line (ambient pressure or supercharging pressure). This is related primarily to the construction of sensors. It is observed in electronical indicators in which recourse piezo-quartz sensors and strain gauge type sensors. In order to minimize this error is constructed signal amplifiers, using a high impedance input. "Flow" line reference pressure has an impact primarily on heat release rate calculated [4, 21-24].

For most of marine engine, cylinder pressure sensor is mounted on the indicator cock. Engine combustion chamber with an indicator cock were combined with the gas tunnel, the length of which depends on the design of the cylinder head and can rise up to several tens of centimeters. The gas channel and indicator cock are the cause of the measured signal distortions and their delay. In research of four-stroke, medium-speed, marine diesel engines [5], has been shown that delays may be several degrees of crankshaft rotation. Signal distortion characteristic of medium-speed engines, are negligible small in two-stroke slow-speed marine engines. If the sensor is mounted in special tunnels of the cylinder head, these types of measurement problems do not occur. Then however, it can occur the phenomenon of thermal shock sensor, formed by the action of the flame on the front face of the sensor. It leads to a distortion, inter alia, of measured changes of pressure. In study [4] this is shown, that small increase of temperature in piezo-quartz sensors for only about $100 \mathrm{~K}$ causes a decrease in the sensitivity of about 3\%. Also decreases the resistance self, for about $10 \%$. In marine diesel engine heavy fuel oil feeding, additional distortions in measurement circuit may arise as a result of fouling carbon deposit sensor.

The important thing for the quality of the indicator diagram is averaging the received multiple charts and their smoothing. In electronic indicators used in operational practice (in ship engine room) is averaged at least 16 charts. In the case of piston engines used in inland transport, it can be said that we can use even more than 30 charts [4]. By averaging the appropriate number of execution indicated it is possible to eliminate inter alia, random vibration of high frequency electrical noise. After averaging chart should be further smoothing, to eliminate distortion - get a smooth and continual graphs $[3,4]$.

Electronic indicator registers the cylinder pressure variation from the relatively low (the beginning of the compression, the beginning of exhalation) less than $1 \mathrm{MPa}$, up to the maximum even about $20 \mathrm{MPa}$. The accuracy of measurement of the pressure instantaneous value affects resolution analog-to-digital converters and their accuracy. The error of quantification particularly affects the measurement of low pressures.

\section{Study of the impact of the error of the TDC location, on the accuracy calculated heat release characteristics}

TDC position errors are a major source errors of determining the mean indicated pressure and thereby greatly influenced by on value of error the maximum value of the net heat release $\left(Q_{\max }\right)$ and the maximum value heat release rate $\left(q_{\max }\right)$.

In order to demonstrate the error of the impact positional TDC, simulation study was conducted in which was used indicated diagrams obtained on a medium-speed four-stroke marine engine type A25/30 (under laboratory conditions) and the low-speed twostroke engine type RTA76, Sulzer company, in the operating of the ship.

Studies in laboratory conditions was carried out on a test bench of the marine engine SULZER type 3 AL25/30. This diesel engine was loaded self-excited synchronous generator type GD8-500-50. This generator is loaded the water resistor, equipped with a tower cooler of water (brine). The test stand is located in the laboratories of the Department of Marine Power Plant Gdynia Maritime University and is shown in Figure 4 and 5 .

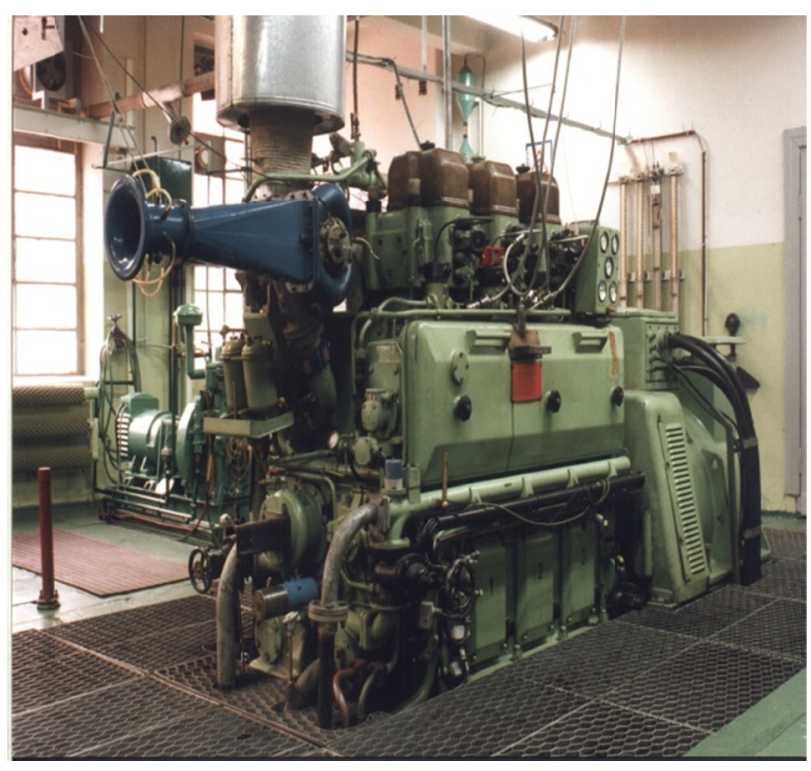

Fig. 4. View of the research stand with the marine diesel engine Sulzer 3AL25/30.

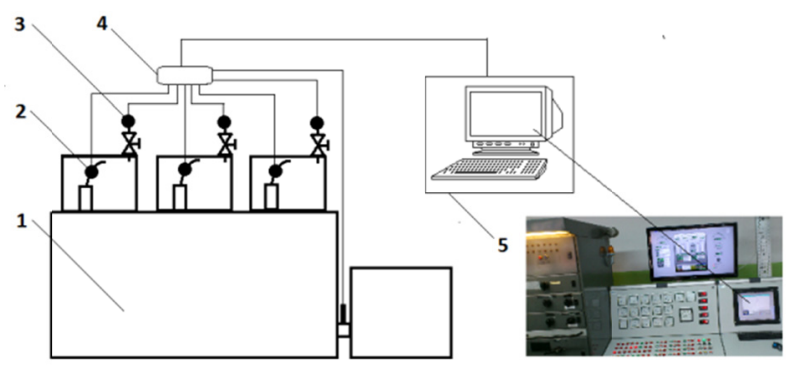

Fig. 5. Scheme of the research stand with the marine diesel engine Sulzer 3AL25/30: 1 - diesel engine Sulzer 3AL25/30, 2 - injection press sensors, 3 - combustion press sensors, 4 - amplifiers and converters $\mathrm{A} / \mathrm{C}, 5$ - computer. 
This stand also includes the electrical switchgear RK73, fuel tanks and a central computer system measuring and registration of operating parameters of the engine. An important element of this measuring system is an electronic indicator UNITEST 2008 in which, to measure the pressure in the cylinders, we use sensors from Kistler 6353A24 with transmitters type 6961A250 (element 1 in Figure 6) and sensors to measure the pressure in the injection system of Optrand type Autopsie-S transducers type C922A8 (element 2 in Figure 6).

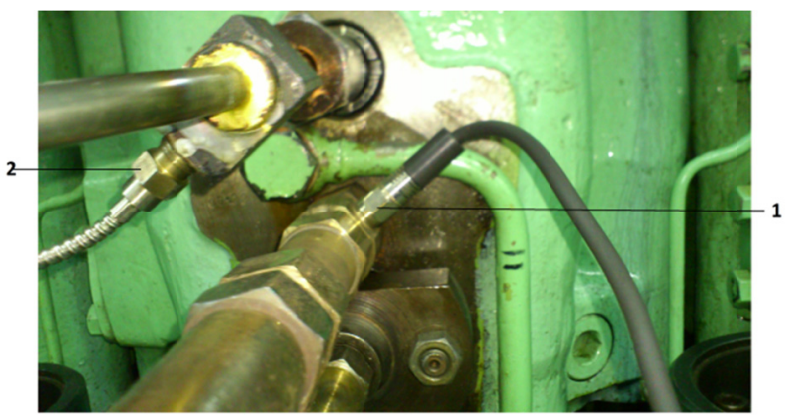

Fig. 6. The research stand with the marine diesel engine Sulzer 3 AL25/30 - view on one of cylinder heads of the mounted sensors: 1 - injection press sensor Optrand typu AutoPSI-S, 2 - combustion press sensor Kistler 6353A24.

Basic specifications of the marine engine SULZER type 3AL25/30:

- power cylinder

- rotation speed

- mean effective pressure

$136 \mathrm{~kW}$,

$750 \mathrm{rpm}$,

$1.575 \mathrm{MPa}$,

- compression ratio

13 ,

- specific fuel consumption

- supercharger system

- turbocharger

$204 \mathrm{~g} / \mathrm{kWh}$,

pulse system,

BBC VTR160N.

For the engine A25/30 it has been adopted TDC location error amounting to $\pm 1^{\circ}$ rotation of the crankshaft and for the low-speed engine RTA76 $\pm 0,5^{\circ}$ rotation of the crankshaft.

In Figures 7 and 8 it has been shown the effect of errors in the TDC location of the indicator diagram on the characteristics of $\mathrm{q}$ and $\mathrm{Q}$ for medium-speed diesel engine (Fig. 5) and low-speed diesel engine (Fig. 6).

Deviations $q_{\max }$ are approximately $\pm 5 \%$ for engine A25 with TDC errors on value $\pm 1^{\circ}$ rotation of the crankshaft and $\pm 3.5 \%$ for the engine RTA76 the TDC with errors ranging $\pm 0.5^{\circ}$ rotation of the crankshaft. $Q_{\max }$ deviations are approximately $\pm 8 \%$ for the A25 engine with TDC errors amounting to $\pm 1^{\circ}$ rotation of the crankshaft and $\pm 4.5 \%$ for the engine RTA76 with TDC errors amounting to $\pm 0.5^{\circ}$ rotation of the crankshaft.

The values of TDC positional errors are intentionally significant. In practice, using the method of determining the TDC based on thermodynamic models, the error should not exceed $\pm 0.3^{\circ}$ rotation of the crankshaft in the case of medium-speed engines and $\pm 0.1^{\circ}$ rotation of the crankshaft in the case of low-speed engines.

It should be noted the almost negligible impact of error TDC on the first phase (the growth steeply runs) $\mathrm{q}$ (Fig. 7, Fig. 8). This is an important observation in the context of the possibility of using of the symptom for diagnostic purposes.

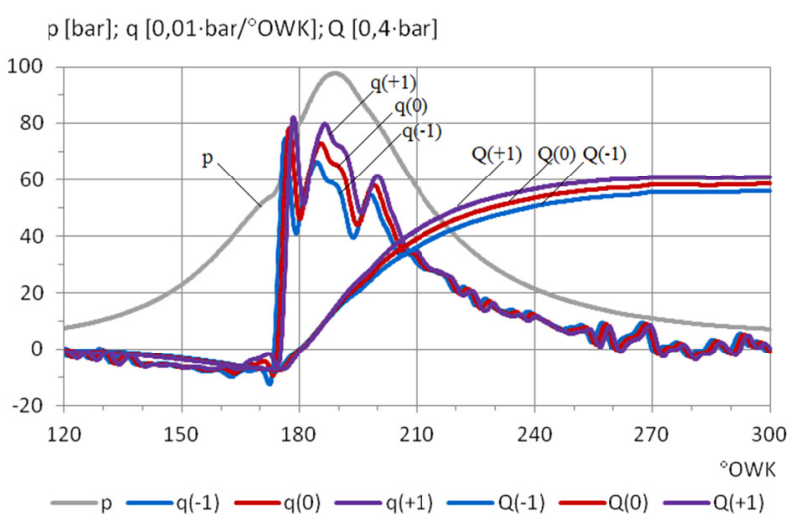

Fig. 7. Effect of TDC position errors on waveforms heat release characteristics $q$ i $Q$ medium-speed marine diesel engine A25/30. The error value TDC (in degrees rotation of the crankshaft) given in brackets.

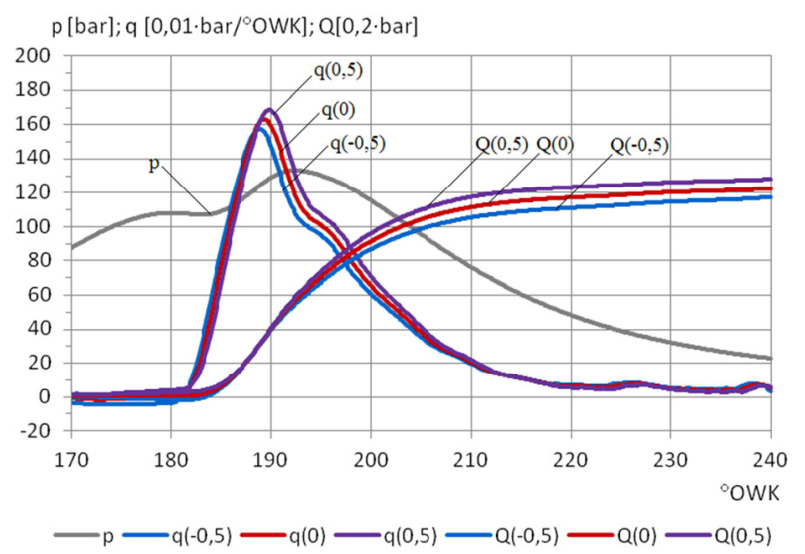

Fig. 8. Effect of TDC position errors on waveforms heat release characteristics $q$ i $Q$ low-speed marine diesel engine RTA76. The error value TDC (in degrees rotation of the crankshaft) given in brackets.

\section{Summary}

The analysis of indicated parameters read from the indicator diagram is now widely use on vessels in the diagnosis of marine engines. The direct analysis of the characteristics feature indicator diagram and changes of indicated parameter values does not always allow on the formulation diagnostic correct conclusions.

Even a few different defects can cause similar changes in the values of indicated parameters and shape indicator diagrams. Therefore this is recommended to conduct in-depth analysis of these graphs based on calculated heat release characteristics.

Measuring systems for electronic indication should meet a number of important requirements to guarantee the possibility of their diagnostic use. These include: high precision sensors for the measurement of cylinder pressure, high speed and accuracy of measuring and recording of measured values, a reliable determination of the top dead center piston (TDC). 
In many cases, in order to obtain a reliable diagnosis is indicated in-depth analysis of these graphs based on calculated heat release characteristics. Then, however, it is essential to cylinder pressure measurement accuracy the accuracy of the indicator diagram, including the exact location of TDC in the diagram.

The analysis of the results indicates that the error location TDC to $1 \%$ results in a significant deviation of the net heat release maximum value and heat release rate, to a few percent (from $3.5 \%$ to $8 \%$ ).

\section{References}

1. S. Polanowski, Journal of Kones (1995)

2. S. Polanowski, Journal of Kones (2000)

3. A. Ambrozik, P. Ambrozik, Analiza i symulacja wykresu indykatorowego tłokowego silnika spalinowego (Szczecin, 2001)

4. A. Ambrozik, Wybrane zagadnienia procesów cieplnych $w$ tłokowych silnikach spalinowych (Kielce, 2003)

5. S. Polanowski, Zeszyty Naukowe Akademii Marynarki Wojennej (2007)

6. A. Ambrozik, Analiza cykli pracy czterosuwowych silników spalinowych (Kielce, 2010)

7. G.M. Szymanski, M. Josko, F. Tomaszewski, R. Filipiak, Mechanical Systems and Signal Processing 58-59, 298-307 (2015), DOI: 10.1016/j.ymssp.2014.12.017

8. G.M. Szymanski, F. Tomaszewski, Proceedings of The 17th International Congress on Sound and Vibration (Cairo, 2010)

9. M. Orczyk, F. Tomaszewski, B. Czechyra, G.M. Szymanski, Proceedings of the International Congress on Sound and Vibration (Athens, 2016)

10. T. Rychter, A. Teodorczyk, Modelowanie matematyczne roboczego cyklu silnika tłokowego (Warszawa, 1990)

11. J.A. Wajand, Pomiary szybkozmiennych ciśnień $w$ maszynach tlokowych (Warszawa, 1974)

12. C. Specht, J. Rudnicki, Polish Maritime Research 23, 20-27 (2016), DOI: 10.1515/pomr-2016-0028

13. D. Pietras, Polish Maritime Research 23, 58-63 (2016), DOI: 10.1515/pomr-2016-0032

14. I. Pielecha, W. Cieslik, Journal of Thermal Analysis and Calorimetry 126, 815-827 (2016), DOI: 10.1007/s10973-016-5544-1

15. I. Pielecha, M. Skowron, W. Bueschke, W. Cieslik, K. Wislocki, IOP Conference Series-Materials Science and Engineering 148, UNSP 012081 (2016), DOI: 10.1088/1757-899X/148/1/012081

16. R. Zadrag, Solid State Phenomena 196, 74-81 (2013), DOI: 0.4028/www.scientific.net/SSP.196.74

17. J. Rudnicki, R. Zadrag, Polish Maritime Research 21, 57-65 (2014), DOI: 10.2478/pomr-2014-0042

18. I. Pielecha, P. Borowski, J. Czajka, K. Wislocki, J. Kazmierowski, Journal of Thermal Analysis and
Calorimetry 119, 695-703 (2015), DOI: 10.1007/s10973-014-4139-y

19. I. Pielecha, K. Wislocki, P. Borowski, W. Cieslik, Journal of Thermal Analysis and Calorimetry 122, 473-485 (2015), DOI: 10.1007/s10973-015-4735-5

20. L.J. Kapusta, I. Pielecha, K. Wisłocki, A. Teodorczyk, Journal of Thermal Analysis and Calorimetry 123, 819-828 (2016), DOI: 10.1007/s10973-015-4927-z

21. M. Skowron, I. Pielecha, K. Wislocki, IOP Conference Series-Materials Science and Engineering 148, UNSP 012085 (2016), DOI: 10.1088/1757-899X/148/1/012085

22. J. Markowski, J. Pielecha, R. Jasinski, T. Kniaziewicz, P. Wirkowski, E3S Web of Conferences 10, UNSP 00140 (2016), DOI: $10.1051 / \mathrm{e} 3$ sconf/20161000140

23. M. Noga, Applied Sciences - Basel 7, 295 (2017), DOI: 10.3390/app7030295

24. H. Golas, A. Mazur, J. Gruszka, ACSR-Advances in Computer Science Research 57, 257-264 (2016) 